\title{
Instability to Longitudinal Vortices in Wavy Shear Flow
}

\author{
W.R.C. Phillips, Q. Shen and Z. Wu \\ Department of Theoretical and Applied Mechanics \\ University of Illinois at Urbana-Champaign \\ Urbana IL 61801-2935, USA
}

\begin{abstract}
Initially spanwise-independent small but finite-amplitude two-dimensional rotational waves and their nonlinear interaction with unidirectional $O(1)$ shear flows are reviewed from the viewpoint of their instability to longitudinal vortex form. The wave-mean interaction is described by a generalized Lagrangian-mean formulation in combination with a separate theory to account for the back effect of the developing mean flow on the wave field. Both the inviscid and viscid eigenvalue problems relevant to the instability are discussed.
\end{abstract}

\section{Introduction}

Nature is replete with shear flows which exhibit both mean and fluctuating parts that subsequently interact to form longitudinal vortices. Examples include Langmuir circulations [12], which occur beneath wind driven surface waves, streamwise rolls in the atmosphere over surface waves [23] or mountainous terrain [13,21]; and quasi-streamwise vortices in laboratory boundary layers (both laminar [10] and turbulent [3]), free shear layers [2] and Stokes layers [8]. Important in each case is an understanding of the nonlinear processes that couple the mean and fluctuating motions, and moreover the secondary and possible tertiary phenomena attributable to the nonlinear rectification of those oscillatory motions: e.g. modifications to the mean flow as a result of the waves and the back effect, if any, of those mean flow modifications on the wave field.

Crucial to such studies are quantities that follow individual fluid particles, quantities alas that are poorly described by the Eulerian equations of mean motion. Indeed, Eulerian-mean vorticity as defined by Reynolds averaging has no simple conservative properties, even when viscosity is ignored and thus acts to conceal the role played by nonlinear rectification (in its guise as Stokes drift) in vortex line deformation.

Andrews and McIntyre [1] sought to define a more rational way to separate 'wave' from 'mean flow' and to define wave-mean interactions; their work culminated in the generalized Lagrangianmean (GLM) equations. These equations describe the back effect of oscillatory disturbances upon the mean state and are exact provided the mapping between the true Lagrangian and the reference GLM remains invertible. Of course GLM still describes mean motions and is therefore conceptually equivalent to Reynolds averaging, but it describes Lagrangian aspects of the motion from an Eulerian framework and is consequently able to capture structural aspects of the flow.

Although unfamiliar to many, and despite difficulties associated with critical layers, the GLM approach appears to be quite fruitful in some classes of problems. The object of the present work is therefore to review one such class of problems: in particular the application of GLM to describe 
mean structures in unidirectional $O(1)$ shear layers (be they viscid or inviscid) owing to the presence of neutral finite-amplitude rotational waves that are initially spanwise independent.

Wave-mean interactions of this ilk were first investigated by Craik [4] and Leibovich [11], who sought to model Langmuir circulations. They considered $O(\epsilon)$ neutral irrotational waves, where $\epsilon$ is the characteristic wave slope, interacting with an $O\left(\epsilon^{2}\right)$ unidirectional Eulerian mean shear flow and found the interaction unstable to longitudinal vortex form via an instability now known as CL2, or Craik-Leibovich type-2. CL2 continues to operate in $O(\epsilon)$ mean shear flows with only minor modifications to the theory, but that is not the case for strong $O(1)$ shear flows, where the back effect of the mean flow modification upon the wave field must be explicitly calculated (Craik [6]). In essence, waves do not drive CL2 (McIntyre and Norton [14]) but act through the pseudomomentum as a catalyst. This means that the magnitude of the mean flow modification is bound not by the strength of the waves but by the magnitude of the pre-existing vorticity in the initial state. With sufficiently strong pre-existing vorticity, therefore, the mean flow modification acts to distort the waves. Of course the detailed kinematics of the instability mechanism are less clear with $O(1)$ shear than with $O\left(\epsilon^{2}\right)$, though the seminal idea of the CL2 instability remains within the theory. In consequence we refer to this form of the instability as CL2-O(1).

The resulting eigenvalue problem for $O(1)$ shear flows in the presence of $O(\epsilon)$ rotational neutral waves is far more complex than its counterpart for weaker shear; requiring inter alia a further differential equation to account for wave distortion. That notwithstanding, Craik [6] was able to construct an inviscid theory and to obtain definite results analytically to demonstrate the existence of longitudinal vortex instability when the spanwise spacing of the vortices is small. This same technique was extended and CL2-O(1) found to operate in a different, wider class of flows by Phillips and Shen [18]. Numerical results by Phillips and $\mathrm{Wu}$ [20] and Phillips et al. [21] concur, and further indicate that wave distortion acts (i), to diminish catalytic action for all but the shortest waves; and (ii), to suppress the instability markedly if the waves are sufficiently long. More recently, Phillips et al. [21] have shown that the CL2-O(1) instability is physically realizable and that the data of Gong et al. [7] represent the first documented observations thereof.

We begin with a brief review of GLM (Section 2) and then specialize the GLM-equations to the problem of $O(\epsilon)$ neutral waves interacting with an $O(1)$ unidirectional shear flow (Section 3). The waves are initially two-dimensional. An appropriate numerical scheme is sketched in Section 4 and detailed results for the case of uniform and nonuniform shear in Section 5. The relevant viscous eigenvalue problem is outlined in Section 6.

\section{The generalized Lagrangian-mean equations}

Andrews and McIntyre's [1] generalized Lagrangian-mean equations are an exact and very general Lagrangian-mean description of the back effect of oscillatory disturbances upon the mean state. The formulation is based upon an exact Lagrangian-mean operator $\left({ }^{-}\right)^{L}$, corresponding to any given Eulerian-mean operator $\left(^{-}\right)$, through an exact disturbance-associated particle displacement field $\xi(\mathbf{x}, t)$ and is valid provided the mapping $\mathbf{x} \mapsto \mathbf{x}+\xi$ is invertible. In consequence dependent variables are given an Eulerian description with position $\mathbf{x}$ and time $t$ as independent variables. The Lagrangian-mean velocity $\overline{\mathbf{u}}^{L}$ is then the velocity field describing trajectories about which the fluctuating particle motions have zero mean, when any averaging process is applied.

For homentropic flows of constant density $\rho$ in a non-rotating reference frame the GLM momentum equation is

$$
\bar{D}^{L}\left(\bar{u}_{i}^{L}-\bar{p}_{i}\right)+\bar{u}_{k, i}^{L}\left(\bar{u}_{k}^{L}-\bar{p}_{k}\right)+\pi_{, i}=-X_{i},
$$

where repeated indices imply summation, commas denote partial differentiation and $\left(x_{1}, x_{2}, x_{3}\right)$ 
$\equiv(x, y, z)$. The operator $\bar{D}^{L}$ is defined as $\bar{D}^{L}=\partial_{, t}+\bar{u}_{j}^{L} \partial_{, x_{j}}$, and the vector wave property $\bar{p}_{i}$, the pseudomomentum per unit mass, is

$$
\bar{p}_{i}=-\overline{\xi_{j, i} u_{j}^{\ell}},
$$

with $\bar{D}^{L} \xi_{j}=u_{j}^{\ell}$. Finally $X_{i}$ are dissipative terms while

$$
\pi=\frac{\overline{\mathcal{P}}^{L}}{\rho}+\bar{\Phi}^{L}-\frac{1}{2}\left\langle u_{j}^{\xi} u_{j}^{\xi}\right\rangle
$$

where $\mathbf{u}^{\xi}$ is the actual fluid velocity and $\Phi^{L}$ is the force potential per unit mass.

We shall restrict attention to fluids in which all mean quantities except possibly the mean pressure $\overline{\mathcal{P}}$ are independent of the streamwise direction; then, with $\Phi^{L}=0, \pi$ reduces to $\overline{\mathcal{P}}^{L}$.

\section{The inviscid interaction of $O(1)$ shear and $O(\epsilon)$ waves}

Craik [6] considered the interaction between an inviscid $O(1)$ primary shear flow and two dimensional straightcrested periodic waves that propagate in (or opposite to) the direction of the flow. Then in a reference frame that moves in the $x$-direction with the phase speed of the waves and with space coordinates $(x, y, z)$, the primary shear flow is $[\bar{u}(z), 0,0]$, where $\bar{u}$ is the Eulerian-mean velocity profile in $\left[z_{B}, z_{T}\right]$. The waves are initially independent of the spanwise coordinate and are of constant amplitude; moreover they induce, in most circumstances, an $O\left(\epsilon^{2}\right)$ pseudomomentum field $\left[\bar{p}_{1}, 0,0\right]$.

Small spanwise-periodic perturbations with streamwise averaged Eulerian velocity components of the form

$$
(\tilde{u}, \tilde{v}, \tilde{w})=\delta \operatorname{Re}\left\{\mathrm{e}^{\sigma t} \mathrm{e}^{\mathrm{i} l y}[\hat{u}(z),-\epsilon \mathbf{i} \hat{v}(z), \epsilon \hat{w}(z)]\right\}
$$

are then envisiaged which, provided the amplitude field of the waves is steady, satisfy continuity correct to $O\left(\epsilon^{2}\right)$ as $l \hat{v}+\hat{w},{ }_{z}=0$. Here $\sigma$ is the growth rate of the spanwise perturbation and $\delta$ is a second small parameter that measures the strength of this motion relative to the primary shear flow; $\delta$ is assumed sufficiently small that linearization with respect to it yields a good approximation to the equations governing the spanwise periodic disturbance. Observe that velocity perturbations in the $y$ and $z$ directions are weaker, by a factor of $\epsilon$, than the $x$ velocity perturbation; this is necessary in order to ensure the GLM equations yield nontrivial solutions for boundary conditions of the form [6] $\hat{w}=0$ at $z=z_{B}, z_{T}$. Accordingly $\sigma=\epsilon \sigma_{1}$.

In such cases the GLM equations reduce to

$$
\begin{gathered}
\sigma_{1} \hat{u}=-\hat{w} \bar{u}^{\prime}, \\
\text { and } \hat{w}^{\prime \prime}+l^{2}\left[\frac{P_{1}^{0^{\prime}} \bar{u}^{\prime}}{\sigma_{1}^{2}}-1\right] \hat{w}=-\frac{l^{2} \bar{u}^{\prime}}{\sigma_{1}} \hat{p}_{1},
\end{gathered}
$$

where prime denotes $d / d z$ and $\bar{p}_{1}$ has been expanded as

$$
\bar{p}_{1}=\epsilon^{2} P_{1}^{0}+\epsilon^{2} \delta \operatorname{Re}\left\{\mathrm{e}^{\sigma t} \mathrm{e}^{\mathrm{i} l y} \hat{p}_{1}(z)\right\}+O\left(\epsilon^{4}, \epsilon^{3} \delta, \epsilon^{2} \delta^{2}\right) .
$$

So $P_{1}^{0}$ is the $O\left(\epsilon^{2}\right)$ component of pseudomomentum [5]

$$
P_{1}^{0}=-\frac{\bar{u}}{2}\left\{\left|\left(\frac{\phi}{\bar{u}}\right)^{\prime}\right|^{2}+\alpha^{2}\left|\frac{\phi}{\bar{u}}\right|^{2}\right\}
$$


in which $\phi(z)$ and $\alpha$ denote the eigenfunction and wavenumber of the primary wave field which together satisfy the Rayleigh equation,

$$
\bar{u}\left(\phi^{\prime \prime}-\alpha^{2} \phi\right)-\bar{u}^{\prime \prime} \phi=0 .
$$

Note that $P_{1}^{0}$ is unbounded in the vicinity of critical layers $(\bar{u}=0)$ unless $\phi \propto \bar{u}^{m}(m \geq 1)$. Such behavior is an indication that the mapping upon which GLM is based has broken down and thus that, except perhaps at a rigid boundary, critical layers should be avoided.

The $O\left(\epsilon^{2} \delta\right)$ spanwise-periodic perturbation of pseudomomentum, $\operatorname{Re}\left\{\mathrm{e}^{\mathrm{i} l y} \hat{p}_{1}\right\}$, arises because the emerging secondary Eulerian velocity field distorts the primary wave field. But because the GLM formulation provides no direct means of evaluating $\hat{p}_{1}$, a separate examination of the wave field is necessary. In carrying out that examination, Craik [6] notes that the $O(\delta)$ spanwise periodic $x$-velocity, $\tilde{u}$, causes the significant part of distortion of the wave field. Then after considerable algebra it follows formally that

$$
\hat{p}_{1}=\mathcal{A}(z) \hat{u}(z)+\mathcal{B}(z) \hat{u}^{\prime}(z)+\operatorname{Re}\left\{\mathcal{C}(z) \hat{\phi}(z)+\mathcal{D}(z) \hat{\phi}^{\prime}(z)\right\},
$$

where $\mathcal{A}, \mathcal{B}, \mathcal{C}$ and $\mathcal{D}$ are functions which are independent of $\sigma$ while $\hat{\phi}(z)$ relates to the $O(\epsilon \delta)$ spanwise periodic wave field modification and satisfies the Rayleigh-Craik equation,

$$
\bar{u}\left[\frac{d^{2}}{d z^{2}}-\left(\alpha^{2}+l^{2}\right)\right] \hat{\phi}-\bar{u}^{\prime \prime} \hat{\phi}=-\hat{u}\left[\frac{d^{2}}{d z^{2}}-\left(\alpha^{2}+l^{2}\right)\right] \phi+\hat{u}^{\prime \prime} \phi .
$$

Thus given the primary Eulerian-mean shear flow $\bar{u}(z)$, the primary wave-field eigenfunction $\phi(z)$ and appropriate boundary conditions, the eigenvalue problem for $\sigma_{1}$ is completely specified by the coupled system (4), (5) and (10) together with (7) and (9).

Craik [6] has further shown that when $l^{2} \gg \alpha^{2}$ and $\alpha=O(1)$ non-trivial solutions to (4), (5) and (10) with homogeneous (i.e. $\hat{\phi}=\hat{w}=0$ on each wall) boundary conditions exist provided

$$
\operatorname{Im}\left\{\int_{z_{1}}^{z_{2}}\left(1+\lambda_{k}\right)^{1 / 2} d z\right\}=\frac{N \pi}{l} \quad(k=1 \text { or } 2)
$$

for some non-negative integer $N$. Here

$$
\begin{gathered}
\lambda_{1,2}=\frac{1}{2}\left\{-\sigma_{1}^{-2}(G+H) \pm\left[\sigma_{1}^{-4}(G+H)^{2}-8 \sigma_{1}^{-2} H\right]^{\frac{1}{2}}\right\} \\
H(z) \equiv \frac{\alpha^{2} \bar{u}^{\prime 2}|\phi|^{2}}{\bar{u}^{2}} \quad \text { and } \quad G(z) \equiv-\alpha^{2} \bar{u}^{\prime}\left(\frac{|\phi|^{2}}{\bar{u}}\right)^{\prime}
\end{gathered}
$$

\section{Numerical solution to the eigenvalue problem}

Galerkin techniques may be used to solve the eigenvalue problem (4), (5) and (10) for the eigenvalue $\sigma_{1}$ which, because (4) and (5) are real, may be real, imaginary or a complex conjugate pair. Complete details are given in Phillips and Wu [20]. Briefly, we suppose $\hat{u}(z)$ and $\hat{\phi}(z)$ can be expanded in linearly independent complete sets of basis functions $u_{i}(z)$ and $\phi_{i}(z)$ as

$$
\hat{u}(z)=\sum_{i=1}^{M} b_{i} u_{i}(z), \quad \hat{\phi}(z)=\sum_{i=1}^{M} b_{M+i} \phi_{i}(z),
$$


where $b_{i}$ and $b_{M+i}$ are the expansion coefficients of $\hat{u}(z)$ and $\hat{\phi}(z)$ respectively, and $M$ is a positive integer; the expansion can be considered exact as $M \rightarrow \infty$. The basis functions were chosen to satisfy the boundary conditions of $\hat{u}$ and $\hat{\phi}$, viz

$$
\hat{u}\left(z_{B}\right), \quad \hat{u}\left(z_{T}\right)=0 ; \quad \hat{\phi}\left(z_{B}\right), \quad \hat{\phi}\left(z_{T}\right)=0 .
$$

Substitution of (14) into (4), (5) and (10) then leads to the residual functions $R_{u}(z)$ and $R_{\phi}(z)$ which must satisfy the inner products

$$
\left\langle R_{u}(z), u_{j}(z)\right\rangle=0 ; \quad\left\langle R_{\phi}(z), \phi_{j}(z)\right\rangle=0 .
$$

A $2 \times M$ order linear eigenvalue problem for $\lambda^{2}$, where $\lambda=l / \sigma_{1}$, results as

$$
\mathcal{L}=\lambda^{2} \mathcal{M}
$$

where $\mathcal{L}$ and $\mathcal{M}$ are $2 M \times 2 M$ order matrices with components that are $M \times M$ order sub-matrices; specifically, the components are functions of $\alpha$ and $l$, but not $\sigma_{1}$.

Of interest is the largest real value of $\sigma_{1}$, or if no real $\sigma_{1}$ exists the complex $\sigma_{1}$ with the largest real part, for each pair $(\alpha, l)$ and the eigenfunctions $\hat{u}$ and $\hat{\phi}$. Chebyshev polynomials were used as basis functions and the accuracy provided by the $M=20$ expansion was considered adequate: to wit, larger $M$ caused no change in the first five significant figures of the importnat eigenvalues. All computations were performed on a DECstation 5000/200 using double precision arithmetic with IMSL routines to solve the eigenvalue problem (15).

\section{Strong inviscid shear between rigid wavy walls}

The criteria for instability (11) requires homogenious boundary conditions. While such boundary conditions can be realized in a variety of situations, it is immediately clear that they are satisfied at rigid wavy walls and as such we shall restrict attention to such cases. We begin in Section 5.1 with the case of uniform shear interacting with two dimensional wavelike disturbances $\alpha \phi$ (which satisfy (8)) of the form $\alpha \phi=\gamma e^{-\alpha z}+\beta e^{\alpha z}$ ( $\gamma, \beta$ constant) assumed initiated by the wavy walls. Note that two very different classes of Rayleigh waves are admissible: those for which $\alpha \phi$ diverges and those for which $\alpha \phi$ converges with $|\alpha z|$ as $|\alpha z| \rightarrow \infty$. Phillips and Shen [18] denote the former 'type-1' and the later, 'type-2' waves. We discuss nonuniform shear in the presence of type-1 and type-2 waves in subsection 5.2.

\subsection{Uniform shear}

In order to proceed analytically, Craik [6] employed (12) and (13) to consider the case $\gamma=0$ and showed that type-1 waves destabilize the flow for $\alpha z \in(0,1)$, as do type-2 waves for all $\alpha z<0$. Phillips and Shen [18] have applied the same technique to other combinations of $\gamma$ and $\beta$. They sought only to determine instability and thus restricted attention to situations which give rise to real $\sigma_{1}$; they do not determine actual values of $\sigma_{1}$. Their findings are plotted in Fig. 1 , in which the shaded portion denotes instability. Note that points of symmetry at $\gamma / \beta= \pm 1$ are clearly evident, as is Craik's result. Note also that $\gamma / \beta=-1$ is stable for all $|\alpha z|$ greater than zero. Interestingly, $\gamma / \beta=-1$ is the only situation that admits, at least in the limit $\alpha z \rightarrow 0$, the amplitude $\alpha \phi \rightarrow 0$, giving rise to what is tantamount to plane Couette flow subject to infinitesimal waves, an inviscid configuration long known to be stable.

But although bounds deduced from (12) and (13) are useful to determine whether the flow is stable or unstable to longitudinal vortex form, these equations alone do not yield the growth rate 
or resolve whether wave distortion acts to enhance or inhibit the instability. Phillips and Wu [20] determined to answer both questions and, because $\gamma=0$ depicts instability over the widest range of $\alpha z$, chose it as the test case. They then considered the problem both with and without wave distortion.

When wave distortion is ignored, only (4) and (5) with $\hat{P}_{1}=0$ need be solved; moreover from (11) the largest upperbound for $\sigma_{1}$ is then seen to occur when

$$
\sigma_{1} \sim \Lambda^{\frac{1}{2}} \mathrm{e}^{-\alpha}\left(1+2 \alpha+2 \alpha^{2}+\frac{4}{3} \alpha^{3}\right)^{\frac{1}{2}} \quad\left(\alpha \neq 0, \quad l^{2} \rightarrow \infty\right)
$$

which is markedly different to the case with wave-distortion, as we see in Fig. 2. Indeed the two cases are asymptotically equivalent only as $\alpha z \rightarrow+\infty$ and it is evident that wave distortion plays an increasingly greater role as $\alpha z$ decreases from $+\infty$.

\subsection{Non-uniform shear}

Phillips and Shen [18] extended the analytical techniques introduced by Craik [6] in the limit $l^{2} \gg \alpha, \alpha=O(1)$ to deduce that type-1 and/or type-2 waves are unstable to longitudinal vortex form in the presence of a wide variety of mean velocity profiles, from boundary layers to mixing layers, which fall within the class $\bar{u}= \pm|z|^{q}$ where $q$ is a real constant. Indeed the implication is that CL2 is ubiquitous to a wide range of wave-mean interactions as we see in Fig. 3. Again the shaded region denotes instability. Moreover following Craik [6], they show in general that small amplitude Rayleigh waves catalyze the instability wherever, in the direction of increasing mean flow, the relative increase in mean flow exceeds the relative increase in wave amplitude.

In order to deduce more detail, several cases of particular physical interest were treated numerically: to wit, an exponentially decaying velocity profile beneath surface gravity waves [20] and power-law and logarithmic-law velocity profiles as would occur in atmospheric boundary layers over wavy terrain [21]. The gross features in each case are much as those in the case of uniform shear, although of course details vary. One point of particular interest, evident by comparing Figs. 2a-b, is that the fastest growth rate occurs when $\alpha=O(1)$ for all $l$ in the presence of rotational waves, in contrast to that for irrotational waves (and uniform shear) where the long wave limit $\alpha \rightarrow 0$ yields the fastest growth rate.

\section{Strong viscid shear between rigid walls}

Although the CL2 instability is inviscid, it can at times be modified by viscosity and the appropriate eigenvalue problem that accounts for viscosity is given by Phillips [17]. Here it is best to work in terms of the associated velocity field $\bar{u}+\bar{d}_{1}-\bar{p}_{1}$, which must be determined as part of the problem; here $\bar{d}$ is the generalized Stokes drift. Indeed the problem comprises two parts: (i), a two-dimensional primary instability composed of equilibrated waves interacting with a mean flow. And (ii), a three dimensional secondary instability, which is manifest as longitudinal vortices, giving rise to a new mean flow often vastly different from its unperturbed laminar forbear. A particularly interesting case is that of periodic Poiseuille flow.

\subsection{Periodic Poiseuille flow}

Phillips [15, 16] and Phillips and Tu [19] consider the development of secondary instabilities via the CL2 mechanism on a nonlinear equilibrium solution in periodic Poiseuille flow. They choose a nonlinear equilibria whose streamwise and spanwise average (of velocity and kinetic energy) 
mimics that of fully developed turbulent channel flow. To proceed, however, they exclude wave distortion, arguing that it should not greatly affect the stability boundary or secondary neutral curve which separates the primary or two dimensional equilibrium from the secondary or three dimensional equilibrium. The neutral curve, defined by the steady states of the eigenvalue problem, is depicted in Fig. 4, which plots the spanwise periodicity of the vortices $\left(L^{+}\right.$, in wall units) as a function of Reynolds number $\left(R e_{\tau}\right.$, based upon channel half width and friction velocity). Further, to compare with Direct numerical simulations, they include the $\mathbf{k}=(0,1,1),(0,2,1)$ and $(0,3,1)$ modes (implying streamwise vortices) calculated by Sirovich et al. [22], along with Jiminez and Moin's [9] minimum width; that is the minimum periodic channel width necessary for turbulence to sustain itself at $R e_{\tau}=180$. All lie close to the first neutral mode. Two features of this mode are striking: first it indicates that (at $R e_{\tau} \approx 90$ ) vortices have a maximum growth rate at spacings roughly twice the preferred 100 wall unit spacing of streaks; and second, that the three dimensional equilibrium collapses near that same preferred spacing. This means that rather than depict the spacing of highest growth rate, streaks mark the smallest spacing at which streamwise vortices occur. Moreover, in addition to the first mode, a higher-order mode is evident that is linearly most unstable at a Reynolds number higher than critical but with a much lower spanwise wavelength, about 40 wall units, a tad more than twice the empirical optimal spacing of riblets, a drag reducing device. Phillips and Tu [19] conjecture that riblets excite this mode of instability.

\section{Summary}

It is shown that the generalized Lagrangian-mean equations are a powerful and useful tool for describing the interaction of rotational finite amplitude waves with shear flows of all orders. Here we restricted our review to $O(1)$ shear flows, as these are common in a variety of geophysical and engineering situations. In such situations two-dimensional waves interact with the mean flow to catalyze an instability - known as Craik-Leibovich type-2 - to longitudinal vortex form. The resulting spanwise periodic secondary flow, however, is sufficiently strong to distort the original wave field and this in turn affects the growth rate of the instability. Results to date indicate that wave distortion acts to inhibit the instability for all but the shortest waves.

Results for both inviscid and viscid interactions are presented, although more emphasis is given to the former case which has been more thoroughly explored.

\section{Acknowledgement}

This work was supported by the National Science Foundation through grants CTS 9008477 and OCE 9503456.

\section{References}

[1] Andrews, D.G. and McIntyre, M.E., "An Exact Theory of Nonlinear Waves on a LagrangianMean Flow," J. Fluid Mech., 89, pp. 609-646, 1978.

[2] Bernal, L.P. and Roshko, A., "Streamwise Vortex Structure in Plane Mixing Layers," J. Fluid Mech., 170, pp. 499-526, 1986.

[3] Corrsin, S., "Some Current Problems in Turbulent Shear Flows," In Symposium on Naval Hydrodynamics (Publn. 515, NAS-NRC, Washington, DC, 1957), pp. 373. 
[4] Craik, A.D.D., "The Generation of Langmuir Circulations by an Instability Mechanism," $J$. Fluid Mech., 81, pp. 209-223, 1977.

[5] Craik, A.D.D., "The Generalized Lagrangian-Mean Equations and Hydrodynamic Stability," J. Fluid Mech., 125, pp. 27-35, 1982.

[6] Craik, A.D.D., "Wave-Induced Longitudinal-Vortex Instability in Shear Layers," J. Fluid Mech., 125, pp. 37-52, 1982.

[7] Gong, W., Taylor, P.A. and Dörnbrack, A., "Turbulent Boundary Layer Flow over Fixed Aerodynamically Rough Two-Dimensional Sinusoidal Waves," J. Fluid Mech., 312, pp. 1-37, 1996.

[8] Hino, M., Sawamoto, M. and Taksu, S., "Experiments on Transition in an Oscillatory Pipe Flow," J. Fluid Mech., 75, pp. 193-207, 1976.

[9] Jiminez, J. and Moin, P., "The Minimal Flow Unit in Near Wall Turbulence," J. Fluid Mech., 225, pp. 213-240, 1991.

[10] Klebanoff, P.S., Tidstrom, K.D. and Sargent, L. M., "The Three-Dimensional Nature of Boundary Layer Instability," J. Fluid Mech., 12, pp. 1-34, 1962.

[11] Leibovich, S., "Convective Instability of Stably Stratified Water in the Ocean," J. Fluid Mech., 82, pp. 561-585, 1977.

[12] Leibovich, S., "The Form and Dynamics of Langmuir Circulations," Ann. Rev. Fluid Mech., 15, pp. 391-427, 1983.

[13] LeMone, M., "The Structure and Dynamics of Horizontal Roll Vortices in the Planetary Boundary Layer," J. Atmos. Sci., 30, pp. 1077-1091, 1973.

[14] McIntyre, M.E. and Norton, W.A., "Dissipative Wave-Mean Interactions and the Transport of Vorticity or Potential Vorticity," J. Fluid Mech., 212 pp. 403-435, 1990.

[15] Phillips, W.R.C., "On the Etiology of Shear Layer Vortices," Theo. Comp. Fluid Dynamics, 2, pp. 329-338, 1991.

[16] Phillips, W.R.C., "The Genesis of Longitudinal Vortices in Free and Bounded Shear Layers," Eddy structure identification in free turbulent shear flows, edited by J. Bonnet and M. Glauser, Kluwer, pp. 35-41, 1993.

[17] Phillips, W.R.C., "On Finite Amplitude Rotational Waves in Viscous Shear Flows," submitted for publication, 1996.

[18] Phillips, W.R.C. and Shen, Q., "On a Family of Wave-Mean Shear Interactions and their Instability to Longitudinal Vortex Form," Stud. Appl. Math, 96, pp. 143-161, 1996.

[19] Phillips, W.R.C. and Tu, H.Y., "Why Riblets Work," Bull. Am. Phys. Soc., 37, pp. 1794, 1992.

[20] Phillips, W.R.C. and Wu, Z., "On the Instability of Wave-Catalysed Longitudinal Vortices in Strong Shear," J. Fluid Mech., 272, pp. 235-254, 1994.

[21] Phillips, W.R.C., Wu, Z. and Lumley, J.L., "On the Formation of Longitudinal Vortices in a Turbulent Boundary Layers over Wavy Terrain," J. Fluid Mech., 326, pp. 321-341, 1996. 


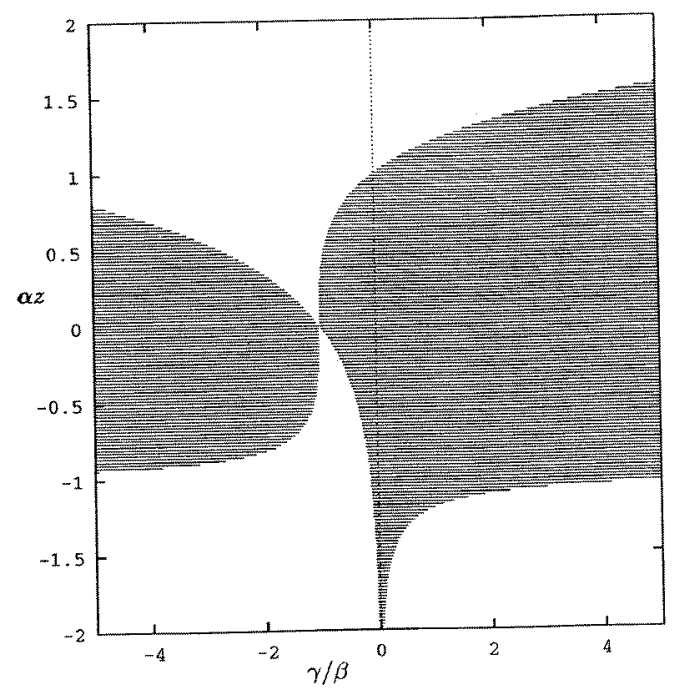

Figure 1: Instability map of $\alpha z$ against $\gamma / \beta$ for uniform shear between rigid wavy walls; shaded region denotes instability ( Phillips and Shen [18]).

[22] Sirovich, L., Ball, K.S. and Keefe, L.R., "Plane Waves and Structures in Turbulent Channel Flow," Phys. Fluids A, 2, pp. 2217-2226, 1990.

[23] Thompson, T.W., Liu, W.T. and Weissman, D.E., "Synthetic Aperture Radar Observation of Ocean Roughness from Rolls in an Unstable Marine Boundary Layer," Geophys. Res. Lett., 10, pp. 1172-1175, 1983. 

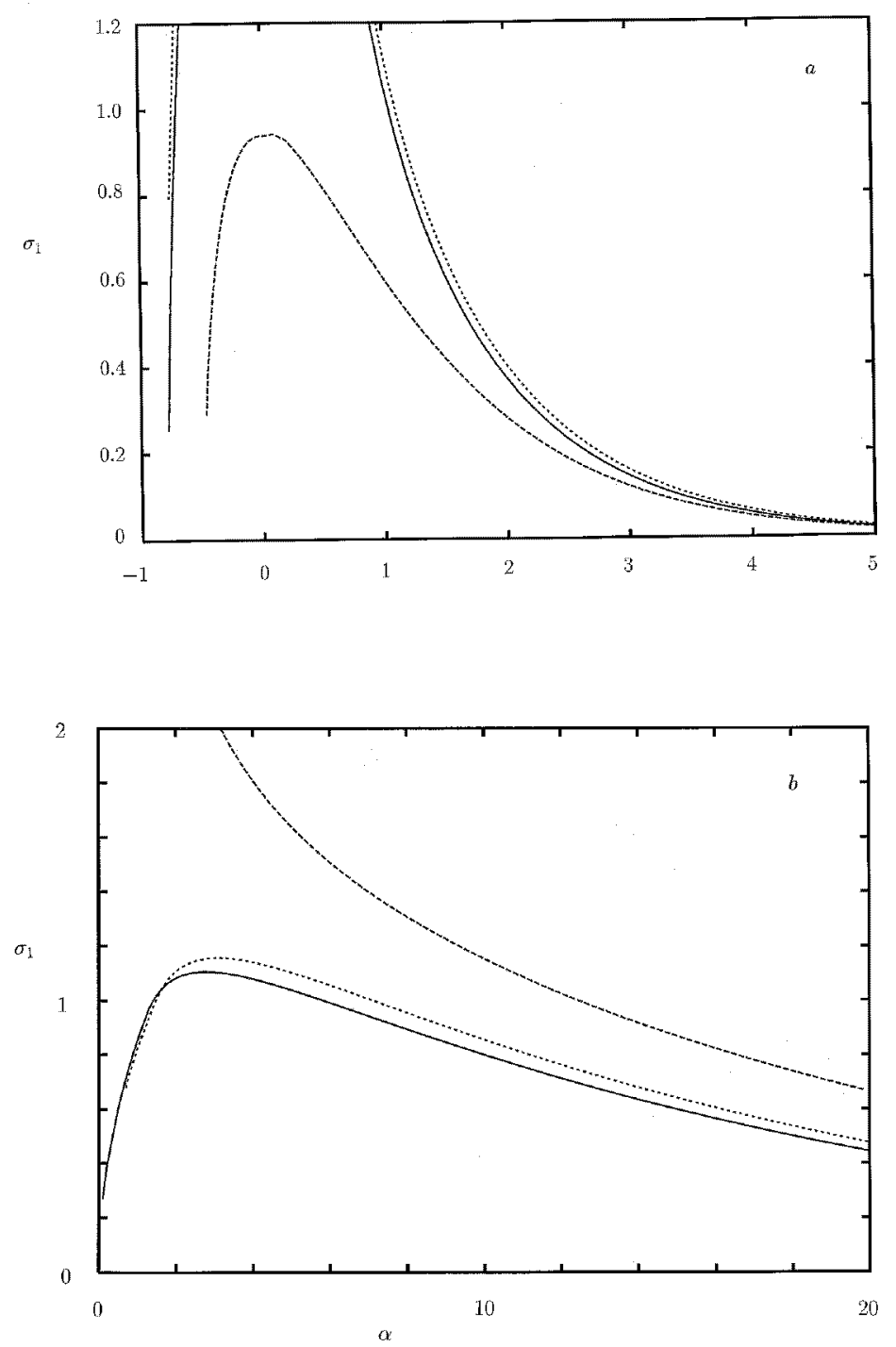

Figure 2: Curves of $\sigma_{1}$ against $\alpha$ in the limit $l^{2} \rightarrow \infty$, both with and without wave distortion: a), uniform shear (Phillips and Wu [20]); b), nonuniform shear (Phillips, Wu and Lumley [21]). 

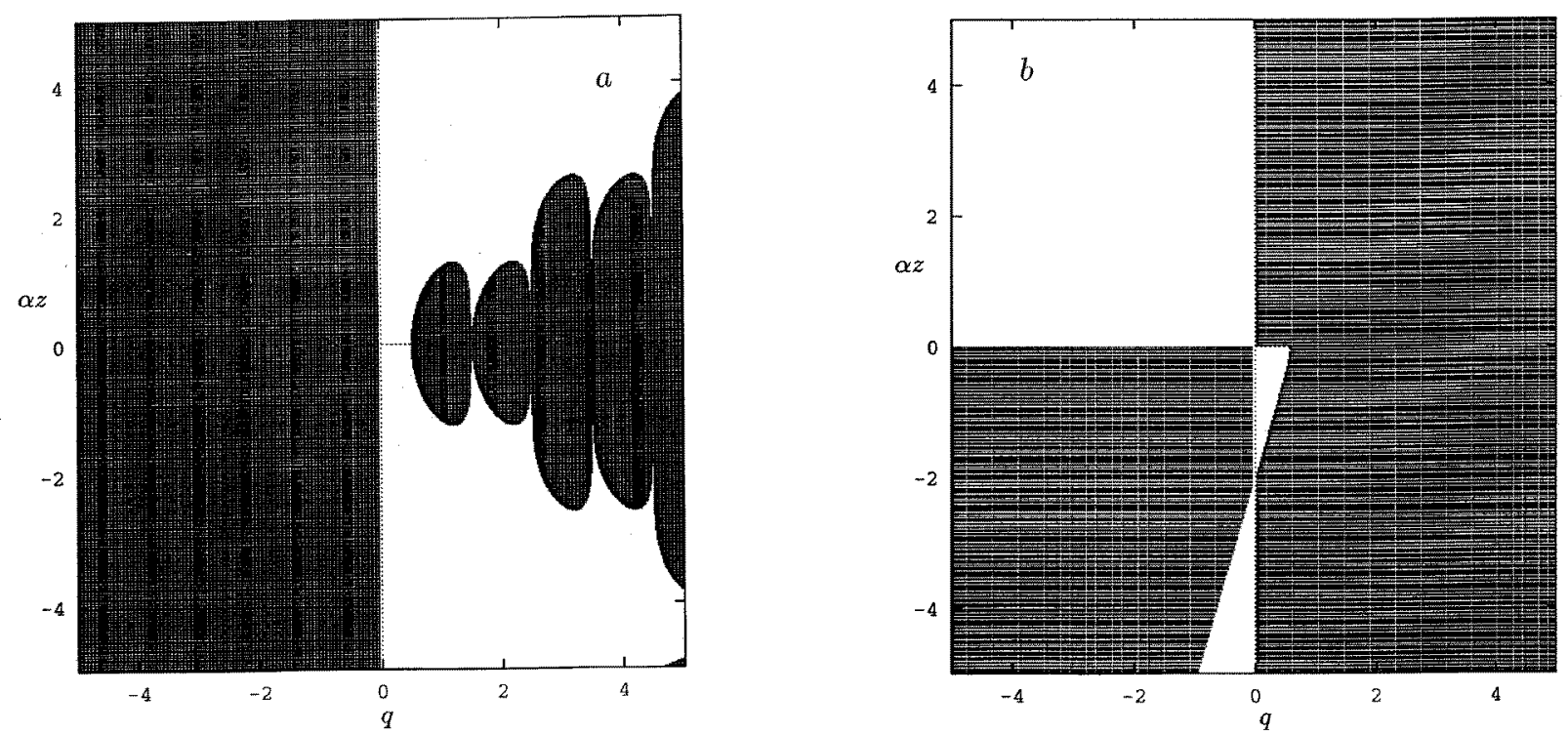

Figure 3: Instability maps for a) type-1 and b) type-2 Rayleigh waves as a function of $q$; instability is denoted by the shaded region (Phillips and Shen [18]).

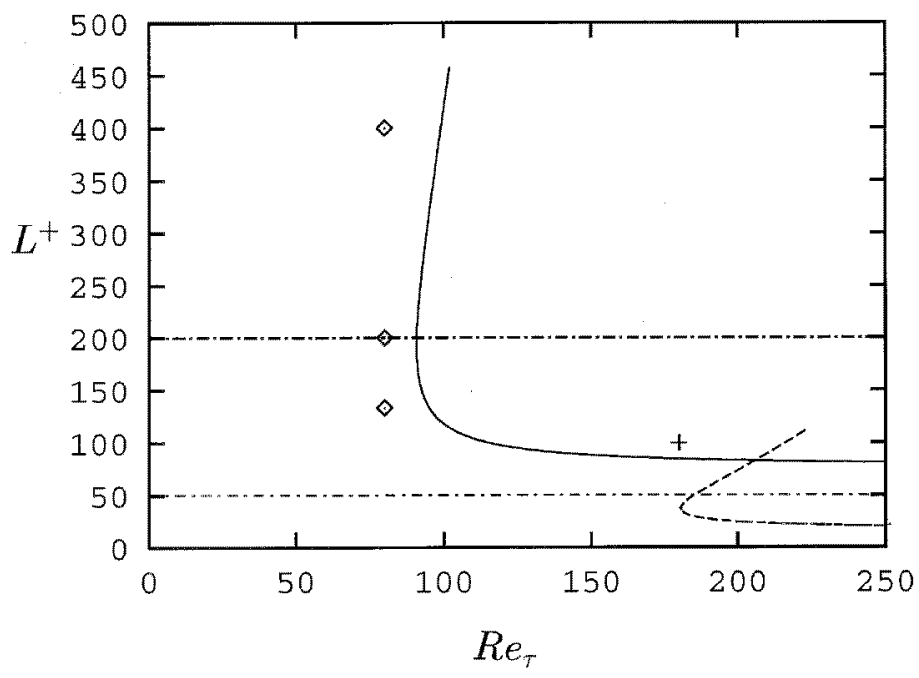

Figure 4: Neutral secondary curves for periodic Poiseuille flow (Phillips and Tu [19], Phillips [16]): $\diamond$, Sirovich, Ball and Keefe [22]; +, Jiminez and Moin[9]. Horizontal lines denote the observed extent of streaks. 